Michael K. Brawer

\section{Prostate Specific Antigen}

Marcel Dekker, New York 2001

328 pp.; USD 150.00

ISBN 0-8247-0555-6

In men, the commonest cancer and a leading cause of death is prostate cancer. One of 8 Americans will develop prostate cancer. Up to $15 \%$ of all males in the USA will have an abnormal prostate specific antigen (PSA) $(<4 \mathrm{ng} / \mathrm{ml})$. Having a serum marker for detection or prognosis is the holy grail of oncologists. Since 1970, PSA has been promulgated as a specific disease marker and increasingly as a screening tool. As a result, an epidemic of prostate cancers has occurred with an $85 \%$ increase in the last decade. Many asymptomatic men have been subjected to prostate biopsies and even radical surgery or radiation. The mortality has also decreased by $14 \%$ in the last decade.

While some point to these changes as evidence for validation of this marker, others argue that the use of PSA has resulted in many insignificant prostate cancers being diagnosed and many men overtreated. These naysayers argue that no long-term randomized study has proven the worth of PSA. Major medical organizations differ greatly in their recommendations for the use of PSA as a screening tool. Those that use the PSA are continuing to define new parameters of the PSA assay including PSA density, velocity, free/bound ratio, etc. Unfortunately, the urological surgical community has embraced the use of PSA and as a result a randomized clinical trial is unlikely to be done soon.

Michael Brawer has collected a series of authors who have written short chapters on many aspects of PSA including the history, chemistry, methods, and variations on PSA measurements (free, total velocity, density, complexes and age specific). Other contributors write about the utility of PSA for detection, repeating the biopsy, staging, and after surgery, radiation, hormonal and chemotherapy. Recommendations are plentiful, but few backup supporting data from controlled randomized trials are presented. There is an excellent chapter that details the various methods and the need to know about how the test has been standardized.

The book represents a complete compendium of information about PSA, primarily from believers in its utility. Comparable data from the skeptics are barely mentioned. No information is presented on the cost effectiveness or utility of PSA testing. The impact on mortality is briefly mentioned. There are occasional statements that are contradictory. One author argues for the use of PSA when a randomized study shows no benefit for that indication. Hopefully, data for evidence-based decisions will be forthcoming. The one area that appears noncontroversial is the use of PSA as an indicator of relapse following treatment.

Paul P. Carbone, Madison, Wisc.

\section{E.R. Barnea, E. J auniaux, P.E. Schwartz (eds)}

\section{Cancer in Pregnancy}

\author{
Springer, Berlin 2001 \\ 310 pp.; CHF 224.70/DEM 261.08/GBP 80.00/USD 139.00 \\ ISBN 1-85233-374-X
}

Cancer is usually a disease of women over 67 years of age, well beyond the reproductive period. However, in women 15-44 years of age, more than 120,000 cancers are diagnosed annually (SEER data). For specific cancers in that population, the proportions of the overall occurrence rates are significant, such as breast (12\%), melanoma $(36 \%)$, thyroid (54\%), cervix (43\%), lymphoma (19\%), and ovary (18\%). These women in the 15 to 44-year age group are likely to have their cancers associated with pregnancies at an estimated rate of 1 in a 1,000 pregnancies. As women delay childbearing, this problem is likely to increase.

For most oncologists and obstetricians this is still a relatively rare problem. However, for the mother and family the association of cancer and pregnancy becomes a nightmare. When it does occur there is a rush to look up the latest data. From personal experience, one is frustrated because individual case reports may not provide a real prospectus on what to do. This book by Barnea, Jauniaux and Schwartz is a tremendous resource document that describes in detail the difficulties and problems faced with managing these patients. The book is divided in two sections. One deals with clinical issues of specific cancers that occur in pregnant women and treatment or diagnostic modalities that are used. The chapters are well illustrated and references are numerous.

The second part of the book deals with biologic issues of the effects of chemotherapy on the fetus and the reproductive potential of the mother. Several interesting chapters go into detail about how the maternal host immune response is able to deal with the foreign tissue of the placenta and the fetus. Other chapters describe how proliferation, differentiation, angiogenesis and placental implantation during pregnancy are likened to cancer growth. Two chapters describe a mechanism that might explain how pregnancy protects against breast cancer through human chorionic growth and luteinizing hormones. A final chapter deals with the ethics and legal issues of the fetus as a patient.

Overall, this small dense book is a very important addition to the literature and should be in the library of all obstetricians and oncologists. For many biologists this volume also brings data that maybe important in understanding how the fetus can survive in an environment that should be lethal to its growth. Most of all we need to remember that the fetus and the placenta are using for growth biologic mechanisms that the cancer cell also exploits. Congratulations to the many international authors who contributed to this book. I hope that future editions can expand on the material in depth.

Paul P. Carbone, Madison, Wisc.

\section{KARGER}

(C) 2002 S. Karger AG, Basel

Fax +4161306 1234

E-Mail karger@karger.ch

www. karger.com
Accessible online at:

www. karger.com/journals/ocl 


\section{Alastair Munro \\ Modern Oncology. An A-Z of Key Topics}

Greenwich Medical Media, London 2001

331 pp.; GBP 22.50

ISBN 1-90015-111-1

Alastair Munro has likened the progress in Oncology to climbing up a 'greased pole'. As soon as you think you have mastered the topic, you slide right back down. Progress in this field is very rapid and the terms used confusing. As a remedy he has prepared a glossary of terms, arranged alphabetically, and accompanied by concise definitions or explanations. The paperback contains definitions from A to $\mathrm{Z}$, starting with abscissa to zing finger protein/motifs. There are a large number of statistical, molecular biological and radiobiological terms explained.

One of the more unusual terms is 'salami publication'. He defines this as a 'reprehensible practice whereby the same data are used in multiple publications'. He describes a situation where the same data are published using completely different authors, making meta-analyses unreliable unless this is recognized. He mentions that a particular analysis of an anti-emetic drug was done where 6 to 11 studies included were duplicates. The conclusions were diametrically opposed if one did the analysis with and without duplicates.

Another term he defines is 'madness among oncologists'. He quotes a recent survey that claimed that about $25 \%$ of oncologists were 'depressed or anxious'. He cites that this is similar to that seen in medical students. No data are known about 'madness' in surgeons. Other interesting terms include 'Inglefinger Rule' and 'Occams Quote'.

This book is intended for the un-initiated in the field as well as those in the field that are exposed to other terms outside their expertise. It is a compact book well worth the investment for libraries of oncologists and cancer researchers. One failing is the lack of references if one wanted to explore any topic further.

Paul P. Carbone, Madison, Wisc.

Barron H. Lerner

\section{The Breast Cancer Wars: Hope, Fear and the Pursuit of a Cure in the Twentieth Century}

Oxford University Press, Oxford 2001

383 pp.; GBP 19.99

ISBN 0-19-514261-6

Breast cancer, a disease known since antiquity, induces fear and hopelessness in those who get the disease which is worsened by the anxiety and pains of treatment with surgery, radiation, and chemotherapy. The damages are both physical and psychological. The author, Barron Lerner, says that these feelings are a result of the public relations efforts of organizations like the American Cancer Society (ACS) that uses their symbol, a sword, and defines the crusade as a war against cancer. In addition, Dr. Lerner feels that women have been subjected to unusual and extensive surgery because of men like Halsted, Hagenson, and Pack who popularized radical mastectomy and even the supraradical. To these medical giants more surgery was better based on biased conclusions.

To write this book, Dr. Lerner conducted many interviews (including myself) to discover the story behind the medical reports in breast cancer. He looked for and found information on the "war against breast cancer'. In particular he describes the efforts of two executives of the ACS, Drs. Cameron and Holleb, who led the public relations efforts of the ACS for many decades and foisted on the American public breast self-examination and mammography, two concepts that have recently been challenged as to their worth. Their pronouncements were not based on evidence or hard data, but assumptions from other screening efforts.

On the other hand, Dr. Lerner puts on a pedestal, men like George Crile and Bernard Fisher, who not only questioned the value of radical mastectomy, but used data and clinical trials to change the paradigms. Dr. Lerner also praises Rose Kushner for her brash but effective way of encouraging women to become involved in the decisions on treatment. Her outspoken methods and the use of political pressure led the way to more funds for breast cancer research and openness about the disease.

This book is a must for all those interested in the history of breast cancer. It is an important historical account of the events of the last century. However, the book is not complete. The important advances in understanding hormonal control of breast cancer and the use of hormones and antihormones in treatment are given few pages. Likewise, the important advances in radiation and chemotherapy that have helped many women are barely mentioned. Hopefully Dr. Lerner will expand on these topics soon. As it is, this book represents an important historical perspective of the issues on the "war on breast cancer' in the 20th century.

Paul P. Carbone, Madison, Wisc.

\section{K. Aziz, G.Y. Wu (eds) \\ Cancer Screening: A Practical Guide for Physicians}

Humana Press, Totowa 2001

324 pp.; USD 89.00

ISBN 0-89603-865-3

Cancer screening aims at detecting cancer early and initiating effective treatment at a reasonable cost, hopefully leading to improved survival. The best and earliest example is Pap test screening for cervical cancer, despite known problems with this test related to quality control or lack of use in certain populations. In other circumstances, screening is applied without adequate evidence-based data to support the application. Costs and behavioral patterns also inhibit the application of other screening tools such as colonoscopy. Drs. Aziz and $\mathrm{Wu}$, University of Connecticut, have gathered experts from around the world to write material for this book, aimed at the practicing physicians. In general, the approach is to describe the epidemiological and causative factors of more than 15 cancers, including liver and esophageal cancers in China and gastric cancer in Japan. Each chapter describes the current screening procedures with a discussion of controversies and ends up with a short summary. There are also two chapters that discuss newer imaging techniques and molecular 
genetic approaches to screening. The information is presented in easy to understand and readable formats. Long lists of data are absent, although each chapter does have a reference list.

I was impressed with the objectivity of most chapters. Prostate and colorectal screening chapters are very clearly done using evidence-based data. The skin cancer screening chapter is very well illustrated with sound recommendations. The breast cancer chapter, on the other hand, is not based on evidence, but recites the current American Cancer Society recommendations that all women over 40 should have annual mammograms. The recommendation for mammography even for women over 50 has been recently challenged. Breast self-examination is considered not to be of any value, except to facilitate more worries among women. The chapters on screening for liver cancer and esophageal cancer in China and gastric cancer in Japan have limited value for US physicians, except for intellectual interest.

Overall, this book is of value for the family physician who wants to have a primer for what to do. He also may be interested in the final chapter that provides medicolegal information on what problems are associated with malpractice suits. Failure to use screening or to follow up on tests is a major cause of succcessful malpractice claims.

Paul P. Carbone, Madison, Wisc.

\section{Richard A. Evans}

\section{The Breakthrough in Cancer You've Never Heard of Your Guide to Limited Surgery in the Treatment of Cancer}

Texas Cancer Center, Houston 2001

375 pp.; USD 16.95

ISBN 0-9708664-0-2

Surgery has been a mainstay for many years and also a major cause of grief. In the first two thirds of the last century many surgeons learned from their mentors that bigger and more surgery was better, resulting in extended radical mastectomy, pelvic exenterations, and forelimb and hind quarter amputations. Because of careful clinical trials, a new paradigm was established during the last third of the 20 th century. Less was found to be as good as radical surgery. Radiation and chemotherapy were found to be not only additive, but also curative, alone and with lesser surgery.

Dr. Evans has written a second edition of his book based on the premise that he needs to popularize the concept of lesser surgery. In addition, he also advocates little or no radiotherapy or chemotherapy. He also offers the concept that local recurrences or lymph nodal disease do not harbinger a poor prognosis. There harbinger is no question, especially in breast cancer, that certain local recurrences can be treated for cure; however, this does not justify a minimalist approach to all recurrences. The author cites a rather biased literature to support his claims and encourages all patients to look for alternatives when offered chemotherapy or surgery. As evidence of his expertise, he cites he is written more than 50 papers, including publications in the New England Journal of Medicine. When his name was searched on Medline, less than 20 references were cited. There were some in the New England Journal of Medicine, but all of these were letters and comments. This was true of most of the others as well. This is hardly a list of publications from an established clinical investigator. Overall, I would have to say that this book has limited value to doctors or patients.

Paul P. Carbone, Madison, Wisc.

\section{R.L. Souhami, I. Tannock, P. Hohenberger, J .C. Horiot}

\section{Oxford Textbook of Oncology, ed. 2}

\section{Oxford University Press, Oxford 2002}

2,852 pp.; GBP 295.00

ISBN 0-19-262926-3

Oncology texts have ranged from small handbooks to large multitome editions. The Oxford Textbook of Oncology can be characterized as multitome ( 2 volumes). There are 20 sections dealing with 170 topics. The textbook is done in small type, increasing the amount of material on its pages. Each section contains numerous tables, charts, and cartoons that add to the text. The overall appearance is one of neatness and consistency attesting to the careful editing of the material. Another characteristic of this textbook is that the authors are predominately non-Americans, providing a different perspective to oncologic topics. The references are very current.

The Oxford Textbook of Oncology is a classic and fresh approach to the field. It is a must for all libraries and all those who like to have a single up-to-date reference book that contains sufficient detail for the clinician in all subspecialties: surgery and medical and radiation oncology. Not only are its clinical chapters outstanding, but the basic science chapters are sufficiently detailed to provide a reference for trainees in the field. The challenge will be to provide updates at regular intervals, as the field of oncology is changing rapidly. A DVD or CD-Rom version would also be helpful.

Paul P. Carbone, Madison, Wisc.

\section{E.R. Barnea, E. J auniaux, P.E. Schwartz (eds)}

\section{Cancer and Pregnancy}

\section{Springer, Berlin 2001}

310 pp.; CHF 224.70, GBP 80.00, USD 139.00

ISBN 1-85233-374-X

Cancer is usually a disease of women over 67 years of age, well beyond the reproductive period. However, in women 15-44 years of age, more than 120,000 cancers are diagnosed annually (Surveillance, Epidemiology, and End Results; National Cancer Institute). For specific cancers in that population, the proportions of the overall occurrence rates are significant such as cancer of the breast $(12 \%)$, melanoma (36\%), thyroid (54\%), cervix (43\%), lymphoma (19\%), and ovary (18\%). These women in the 15 - to 44 -year age group are likely to have their cancers associated with pregnancies at an estimated rate of 1 in a 1,000 pregnancies. As women delay childbearing, this problem is likely to increase. 
For most oncologists and obstetricians this is still a relatively rare problem. However, for the mother and family the association of cancer and pregnancy becomes a nightmare. When it does occur there is a rush to look up the latest data. From personal experience, one is frustrated because individual case reports may not provide a real prospectus on what to do. This book by Barnea, Jauniaux and Schwartz is a tremendous resource document that describes in detail the difficulties and problems faced with managing these patients. The book is divided in two sections. One deals with clinical issues of specific cancers that occur in pregnant women and treatment or diagnostic modalities that are used. The chapters are well illustrated and references are numerous.

The second part of the book deals with biologic issues of the effects of chemotherapy on the fetus and reproductive potential of the mother. Several interesting chapters go into detail about how the maternal host immune response is able to deal with the foreign tissue of the placenta and the fetus. Other chapters describe how proliferation, differentiation, angiogenesis and placental implantation during pregnancy are likened to cancer growth. Two chapters describe a mechanism that might explain how pregnancy protects against breast cancer through human chorionic growth and luteinizing hormones. A final chapter deals with the ethics and legal issues of the fetus as a patient.

Overall, this small dense book is a very important addition to the literature and should be in the library of all obstetricians and oncologists. For many biologists this volume also brings data that may be important in understanding how the fetus can survive in an environment that should be lethal to its growth. Most of all we need to remember that the fetus and the placenta are using growth biologic mechanisms that the cancer cell also exploits. Congratulations to the many international authors who contributed to this book. I hope that future editions can expand on the material in depth.

Paul P. Carbone, Madison, Wisc.

M.H. Steinberg, B.G. Forget, D.R. Higgs, R.N. Nagel

\section{Disorders of Hemoglobin}

\section{Genetics, Pathophysiology, and Clinical Management}

\section{Cambridge University Press, Cambridge 2001}

1268 pp.; USD 275.00

ISBN 0-521-92268-2

If there is any one protein whose study has served as the springboard for investigation of structure-activity relationships, ligandbinding kinetics, protein assembly, and molecular biology in general, it is hemoglobin. This was the first protein for which an amino acid substitution was linked to a specific disease (sickle cell anemia, 1956). Likewise, study of the hemoglobinopathies and the thalassemias has identified not only a sweeping array of molecular mechanisms underlying these disorders but has provided major insights and paradigms for molecular biology in general. The fact that a book of this size and scope is devoted to a single protein is an indication of the wondrous complexity of biology and a harbinger of what still lies ahead.

This reference work, a compilation of remarkably up-to-date review articles by a cast of 53 authors, nearly all major contributors to the field, is the legitimate successor to the two earlier volumes on hemoglobin by Bunn, Forget, and Ranney (1977) and Bunn and Forget (1986). The initial ten chapters cover molecular biology, ontogeny, synthesis, structure and function of hemoglobin. This section begins with an instructive piece by Helen Ranney, which describes the many seminal contributions to scientific knowledge that comprise the history of hemoglobin. The chapter by Max Perutz, the Nobelist who defined hemoglobin structure by crystallography, beautifully summarizes current knowledge on the relationship of structure to function, much of which is due his lifetime of work. Several other chapters are detailed treatises covering the expanding frontier of regulation of gene expression. The next 32 chapters deal exhaustively with the thalassemias and the sickling disorders - molecular biology, genetics, epidemiology, pathophysiology, principles of treatment, and current research frontiers including, e.g., recombinant hemoglobins and engineering of mice with endogenous globin production inactivated and replaced with human sickle hemoglobin production. The chapters on pathophysiology of thalassemia and the sickling disorders are superb reviews. The volume ends with three chapters on other inherited disorders affecting hemoglobin function and the acquired hemoglobin disorders.

This book is an essential resource for anyone with a serious investigational interest in hemoglobin. Likewise, it is clearly the major reference for faculty who teach in some depth about any aspect of hemoglobin. It is also a key, if occasional, source for practicing oncologists and hematologists asked to diagnose and manage any of the less common hemoglobinopathies and thalassemias; here, for example, one can read about what to expect in a patient with compound heterozygosity for hemoglobin $\mathrm{E}$ and $\beta$-thalassemia.

As is not uncommon in multi-authored works of this sort, there is a considerable degree of redundancy between authors. This is true throughout much of the book, from control of gene expression to pathophysiological mechanisms to disease manifestations. And since many chapters tackle the forefront of research, one can find controversial assertions that will require years of work before the criterion of reasonable certainty is met. On the whole, however, it is a superb exposition of current knowledge, and I am very happy to have a copy on my shelf.

Robert D. Woodson, Madison, Wisc.

Carol K. Redmond, Theodore Colton (eds)

\section{Biostatistics in Clinical Trials}

\section{Wiley, New York 2001 \\ 522 pp.; USD 195.00}

ISBN 0-471-82211-6

The topics include history, trial designs, analysis and methods, ethics, short descriptions of trial organizations and a short review of pharmaceutical industry concerns.

Perusal of the table of contents indicates an eclectic collection of topics such as Cochrane Collaboration, European Federation of Statisticians in the Pharmaceutical Industry (EFPSI), and Society for Clinical Trials, just to name a few, in addition to the standard fare covered in texts on clinical trial methodology, but treated briefly and lightly. Also there seems to be uneven treatment of topics. For exam- 
ple, the concept of randomization which forms the fundamental basis of statistical inference in comparative clinical trials is covered in mere four paragraphs and in less than a page. Perhaps that is what one can expect from an encyclopedic volume like this. However, because of its very nature, it covers a number of topics not often covered in typical clinical trials and biostatistics literature such as the history of clinical trials, organizations for clinical trials, ethics, audits and quality control, data management, pharmaceutical industry, and the list goes on.

The material contains a limited amount of mathematical equations and focuses mainly on concepts, both statistical and non-statistical, in mainly descriptive chapters. Most of the articles are written at a level that does not require statistical sophistication of the readers. Each entry in the volume introduces the statistical concepts and other related issues involved in the topic along with a relatively extensive list of references on the topic. As such, this volume can obviously be used as a source material for other texts for classroom teaching. For the clinical investigator who wants to learn about the details, other resources are needed such as the text by Friedman, Furberg and DeMets (1999). It would serve well as a valuable reference and an excellent resource to add to classroom and other teaching tools, but certainly not as a text.

The involvement of biostatisticians in clinical trials does not begin with the analysis of data already generated, but rather in the genesis, design and implementation of a protocol. The biostatistician also becomes a key player in the monitoring and even the ethics of a trial. This latter component involves an independent data monitoring committee for which the biostatistician provides a comprehensive analysis of the recruitment, randomization, toxicities, compliance and data outcomes. Much depends on the outcome of the trial and the validity of its results.

Redmond and Colton have assembled a 522-page volume that is organized like an encyclopedia, alphabetically by topic. The contents are derived from the 'Encyclopedia of Biostatistics' articles (updated and revised) as well as new material for approximately $25 \%$ of the volume.

Paul P. Carbone, KyungMann Kim, Madison, Wisc.

Tai-Ping D. Fan, Elise C. Kohn (eds)

\section{New Angiotherapy}

Humana Press, Totowa 2001

USD 155.00

ISBN 0-896-3464-X

Since the 1950s after the initial publication of Judah Folkman, there has been a long series of publications that have investigated angiogenesis. In the laboratory, fundamental discoveries have been made that define scores of substances that both stimulate or inhibit new blood vessel formation. More recently, some of the biologic products have actually reached clinical trials. The excitement was followed by a long drought of discoveries. However, the past 5 years have seen an explosion of possibilities. Angiogenesis and vasculogenesis are now recognized as important biologic phenomena in normal physiological conditions (embryogenesis, implantation and wound healing) as well as abnormal disease states (cancer, rheumatoid arthritis, atherosclerosis and macular degeneration). The laboratory findings have led to hope that these disease states maybe alleviated by application of these inhibitory or stimulatory molecules.

Fan and Kohn have assembled a group of international experts who have written up-to-date and detailed chapters on all aspects of the processes. The term 'therapeutic angiogenesis' encompasses the use of angiogenesis stimulation to overcome certain disease states. The material is quite detailed and aimed at investigators, but the book as a whole is an excellent reference for anyone interested in the field.

The clinical trials for angiotherapy are still in their infancy. The major uses are: (1) What dose to use? (2) How does one monitor the effect? (3) In what clinical environment should they be used? (4) Should these factors be used alone or in combination with other factors or with cytotoxic agents? and (5) What surrogate markers are valid? From a biologic perspective with so many factors, redundancy is very likely to occur. This book is a must reading for the laboratory and clinical investigator in this field.

Paul P. Carbone, Madison, Wisc.

\section{Arthur M. De Schepper (ed.) \\ Imaging of Soft Tissue Tumors, ed. 2}

Springer, Berlin 2001

469 pp.; 955 fig.; 32 tab. (hardcover); USD 199.00

ISBN 3-540-41405-3

\author{
T. Rand, P. Ritschl, S. Trattnig et al.
}

\section{Imaging of Bone and Soft Tissue Tumors: A Case Study Approach}

Springer, Berlin 2001

166 pp.; 172 fig. (softcover); USD 69.00

ISBN 3-540-65096-2

Imaging of Soft Tissue Tumors is intended as a reference guide for clinicians looking for an optimal approach to the difficult subject of soft tissue tumor evaluation. The target audience for this textbook includes specialists such as oncologists and radiologists seeking a better grasp of a topic that has not received much attention.

This comprehensive book is thoughtfully organized into four sections: 'Imaging Modalities', 'Staging, Characterization, and Grading', 'Imaging of Soft Tissue Tumors', and 'Imaging after Treatment'. The first section commendably explains and demonstrates the key concepts of various imaging modalities, such as ultrasound, plain radiography, angiography, $\mathrm{CT}$, nuclear medicine, MRI and dynamic contrast-enhanced MRI. The inclusion of color images, notably in the Doppler ultrasound section, is a pleasant surprise. The staging and grading section provides a detailed explanation of the difficulty in differentiating benign from malignant soft tissue tumors, particularly with MRI. The incorporation of multiple tables reinforces the key concepts discussed. The third section deals with an assortment of tumors organized by histologic type. Each tumor section provides a succinct discussion of the disease and incorporates high quality images. This section is further enhanced by a discussion of biopsy techniques and the inclusion of rare tumor images. The final section 
on imaging after treatment briefly discusses the MRI features of treated tumors and the more advanced topic of dynamic contrastenhanced MRI. Dynamic MRI has received increased attention because it is a useful adjunct in assessing treatment response and recurrence.

The high quality of both the paper and prints further enhances this well organized compendium. However, a few shortcomings remain. The presence of a few typographical and grammatical errors in the text and figures does not weaken its worth, but they are unexpected for a second edition. Some readers may find the writing style too rigid and 'textbook-like', making for a slow read. This is especially apparent in the MRI physics section, which is slightly awkward and may be too technical for a nonradiologist.

The second book, Imaging of Bone and Soft Tissue Tumors, presents 39 real-life cases collected from the authors' grand rounds.
The cases are in random order, uncategorized and cover both common and rare lesions. Each case is divided into four sections, history, imaging findings, differential diagnosis and final diagnosis with succinct disease description. Cases include relevant, high-quality images. The case discussion at the end is concise and covers the basic concepts. Several cases would benefit from further elaboration. The thoughtful attention paid to the layout makes this book easy to use as a self-assessment tool. Overall, the print and image quality is superb and an excellent companion to the Imaging of Soft Tissue Tumors.

In summary, both books are well written, elucidating the various aspects of soft tissue and osseous tumor evaluation. The Imaging of Soft Tissue Tumors book is a useful reference for practicing primary physicians and radiologists. The case study book will help to further test the reader's knowledge about soft tissue and osseous tumors.

James J. Choi, MD 